\title{
1 Genome features of common vetch (Vicia sativa) in natural habitats
}

2 Running title: The genome of common vetch

3

4 Kenta Shirasawa $^{1^{*}}$, Shunichi Kosugi ${ }^{1 \dagger}$, Kazuhiro Sasaki ${ }^{2 \ddagger}$, Andrea Ghelfi ${ }^{1}$, Koei Okazaki ${ }^{1}$, Atsushi

5 Toyoda $^{3}$, Hideki Hirakawa ${ }^{1}$, Sachiko Isobe ${ }^{1}$

6

$7 \quad{ }^{1}$ Kazusa DNA Research Institute, Kisarazu, Chiba 292-0818, Japan, ${ }^{2}$ Institute for Sustainable

8 Agro-ecosystem Services, Graduate School of Agricultural and Life Sciences, The University of

9 Tokyo, Nishitokyo, Tokyo 188-0001, Japan, and ${ }^{3}$ National Institute of Genetics, Mishima, Shizuoka

10 411-8540, Japan

11

12 'Present address: RIKEN, Yokohama, Kanagawa 230-0045, Japan

13 Present address: Japan International Research Center for Agricultural Sciences, Tsukuba, Ibaraki

14 305-8686, Japan

15

16 *Corresponding author: Kenta Shirasawa (shirasaw @ kazusa.or.jp)

17 Tel. +81-438 523935

18 


\section{Highlight}

20 Sequence analysis of the common vetch (Vicia sativa) genome and SNP genotyping across natural

21 populations revealed nucleotide diversity levels associated with native population environments.

\section{Abstract}

24 Wild plants are often tolerant to biotic and abiotic stresses in their natural environments, whereas

25 domesticated plants such as crops frequently lack such resilience. This difference is thought to be

26 due to the high levels of genome heterozygosity in wild plant populations and the low levels of

27 heterozygosity in domesticated crop species. In this study, common vetch (Vicia sativa) was used as

28 a model to examine this hypothesis. The common vetch genome $(2 \mathrm{n}=14)$ was estimated as $1.8 \mathrm{~Gb}$

29 in size. Genome sequencing produced a reference assembly that spanned $1.5 \mathrm{~Gb}$, from which 31,146

30 genes were predicted. Using this sequence as a reference, 24,118 single nucleotide polymorphisms

31 were discovered in 1,243 plants from 12 natural common vetch populations in Japan. Common vetch

32 genomes exhibited high heterozygosity at the population level, with lower levels of heterozygosity

33 observed at specific genome regions. Such patterns of heterozygosity are thought to be essential for

34 adaptation to different environments. These findings suggest that high heterozygosity at the

35 population level would be required for wild plants to survive under natural conditions while allowing

36 important gene loci to be fixed to adapt the conditions. The resources generated in this study will

37 provide insights into de novo domestication of wild plants and agricultural enhancement.

38

39 Keywords: Common vetch; ddRAD-Seq; Genome sequence; Natural populations; Nucleotide

40 diversity; Single nucleotide polymorphism 


\section{Introduction}

43 Wild plants, including weeds that have not yet been domesticated or cultivated, generally possess

44 characteristics that allow them to survive and propagate in their natural environments when

45 challenged by local biotic and abiotic stresses (Mammadov et al., 2018). The resilience exhibited by

46 wild plants is thought to be due to their high levels of genetic heterogeneity (Canc $\square$ ado, 2011).

47 Indeed, genetic heterogeneity was effective in suppressing disease when populations of genetically

48 diversified crops were planted together in the same fields (Zhu et al., 2000).

49 In contrast with wild plants, crop plants have lost their natural survival traits as a result of the

50 extremely low levels of genetic heterogeneity found in monoculture crop species (Mundt, 2002).

51 Therefore, disease-, insect-, and weed-controls are essential in commercial crop cultivation to reduce

52 losses and maximize yields. This requires additional crop management costs for farmers, for example,

53 for labor and agrochemicals. There are two main reasons for the low genetic heterogeneity in crop

54 species. One reason is crop domestication (Izawa et al., 2009), in which only a few plants possessing

55 desirable phenotypes, such as large fruit size, non-seed shattering, and long-seed dormancy, are

56 selected from the broad genetic pools of wild plants. The second reason is selective breeding for

57 desirable traits. While valuable for stabilizing crop phenotypes such as yield, these selective

58 processes have reduced genetic diversity in monoculture crops by purging diverse germplasms $(\mathrm{Fu}$,

59 2015). During domestication and selective breeding, small numbers of alleles that have large effects

60 on phenotypic variations have often been targeted, further reducing the genetic diversity within

61 cultivated varieties (Fernie and Yan, 2019).

62 While remaining more diverse than crop species, wild plant populations have also experienced

63 loss of genetic heterogeneity at some loci, though in wild plants this is due to directional selection

64 and genetic drift. For example, natural populations of Arabidopsis have lost genetic heterogeneity at

65 flowering loci to synchronize flowering time (Mendez-Vigo et al., 2011), which is beneficial for

66 propagation under natural conditions. This suggests that genome-wide genetic heterogeneity is not

67 necessarily required for wild plant populations and that small numbers of loci could become fixed 
68 under certain selective conditions. This suggests that it would be possible to generate new plant

69 populations with a) fixed domestication loci with suitable alleles for agricultural traits and b) high

70 general levels of genetic diversity elsewhere in the genome. Such plant populations could be used as

71 crop species, as proposed by Litrico and Violle (Litrico and Violle, 2015), and would possess natural

72 resistance and suppression traits, as a result of high heterogeneity, that would enhance population

73 resilience to biotic and abiotic stresses. As favorable agricultural alleles would be fixed, the benefits

74 of genetic heterogeneity would exist alongside desirable agricultural traits. Mixtures of heterozygous

75 plant populations have already been used as crops in allogamous species such as onion and clover.

76 However, the potential benefits of genetic heterogeneity for autogamous plants such as legumes

77 remain unclear.

78 Common vetch (Vicia sativa), a wild legume commonly found in open fields, was partially

79 domesticated and cultivated in the past (Bryant and Hughes, 2011). Common vetch therefore has

80 crop potential and can serve as a model for examination of genetic heterogeneity and domestication.

81 The first step is to evaluate the levels of genetic heterogeneity in wild common vetch populations.

82 However, no genome sequence data is available in common vetch. At least three different

83 chromosome numbers $(2 \mathrm{n}=10,12$, and 14) have been reported (Ladizinsky, 1998; Ladizinsky and

84 Waines, 1982). In this study, a reference sequence for common vetch was developed and single

85 nucleotide polymorphism (SNP) analysis with double-digest restriction-site associated DNA

86 sequencing (ddRAD-Seq) was used to evaluate heterogeneity in genomes of common vetch

87 populations.

\section{Materials and methods}

\section{$90 \quad$ Plant materials}

91 A standard inbred line of common vetch ( $V$. sativa), KSR5, was established from a wild plant

92 collected from Kisarazu, Chiba, Japan, by self-pollination for more than three generations. KSR5

93 was used for genome and transcriptome sequencing analysis. For genetic diversity analysis, 1,243 
94 plants were collected from 12 locations across the latitude from $31.3^{\circ} \mathrm{N}$ to $38.8^{\circ} \mathrm{N}$ in Japan (Figure 1 ,

95 Supplementary Table S1). In addition, eight accessions from France, Germany, Greece, Iran, Italy,

96 and Tunisia were obtained from the NIAS Genebank, Tsukuba, Japan (Supplementary Table S1).

97 Genomic DNA was extracted from young leaves with a DNeasy Plant Mini Kit (Qiagen, Hilden,

98 Germany).

Chromosome observation

101 Root tips of two-day-old seedlings of KSR5 were treated with $0.05 \%$ colchicine for 18 hours, fixed

102 with 1:3 acetate:ethanol for 2 hours, and washed three times with water. Cell walls of the root tips

103 were digested with 2\% cellulase (SERVA Electrophoresis GmbH, Heidelberg, Germany), $2 \%$

104 macerozyme (SERVA Electrophoresis $\mathrm{GmbH}$ ), and $0.1 \mathrm{M}$ sodium acetate for four hours at $37^{\circ} \mathrm{C}$.

105 The root tip cells spread on a slide glass were fixed again with 1:3 acetate:ethanol and dried at room

106 temperature. Chromosomes were stained with $1 \mathrm{ug} / \mathrm{mL}$ DAPI (4,6-Diamidino-2-phenylindole) in

107 Fluoro-KEEPER Antifade Reagent (Nacalai Tesque, Kyoto, Japan) and were observed under a

108 confocal laser scanning microscope, LSM700 (Carl Zeiss, Oberkochen, Germany). Chromosome

109 length was measured with ImageJ (Schneider et al., 2012).

111 Sequencing analysis of the common vetch genome

112 Genomic DNA from KSR5 was used to construct one paired-end (insert size of $500 \mathrm{bp}$ ) and four

113 mate-pair sequencing libraries (insert sizes of 2, 5, 10, and $15 \mathrm{~kb}$ ) in accordance with manufacturer

114 protocols (Illumina, San Diego, CA, USA). Libraries were then sequenced using a HiSeq2000

115 instrument (Illumina). A long insert library for KSR5 was also prepared and sequenced on an RSII

116 instrument (PacBio, Menlo Park, CA, USA). The paired-end sequence reads were used for genomic

117 size estimation based on $k$-mer frequency $(k=17)$ using Jellyfish (Marcais and Kingsford, 2011).

118 The paired-end and mate-pair reads were assembled and scaffolded with SOAPdenovo2 (Luo et al.,

119 2012). Gaps, represented by Ns in the scaffold sequences, were filled by PBjelly (English et al., 
120 2012) with PacBio reads, in which sequence errors were corrected with the paired-end reads by

121 proovread (Hackl et al., 2014). Contaminated sequences were removed by BLASTN search

122 (Altschul et al., 1990), with an E-value cutoff of $1 \mathrm{E}-10$ and length coverage of $\geq 10 \%$, against

123 sequences from potential contaminating resources such as organelles (the plastid and mitochondrion

124 genome sequences of L. japonicus and V. faba: KF042344, AP002983, JN872551, and KC189947),

125 bacteria and fungi (NCBI bacteria and fungi), human (hg19), and artificial sequences (UniVec and

126 PhiX). The resulting sequences that were $\geq 1,000 \mathrm{bp}$ in size were selected and designated VSA_r1.0

127 as a draft common vetch genome. Completeness of the assembly was assessed with sets of a

128 Benchmarking Universal Single-Copy Orthologs (BUSCO) (Simao et al., 2015).

RNA sequencing and assembly

131 Total RNA was extracted from ten tissue samples (roots, seedlings, stems, apical buds, immature and 132 mature leaves, tendrils, flower buds, flowers, and pods) using an RNeasy Mini Kit (Qiagen) and

133 treated with RQ1 RNase-Free DNase (Promega, Madison, WI, USA) to remove contaminating

134 genomic DNA. RNA libraries were constructed in accordance with the TruSeq Stranded mRNA

135 Sample Preparation Guide (Illumina). Nucleotide sequences were obtained with a MiSeq instrument

136 (Illumina) in the paired-end 301 bp mode. Low-quality reads were removed using PRINSEQ

137 (Schmieder and Edwards, 2011) and adapter sequences were trimmed with fastx_clipper (parameter,

138 -a AGATCGGAAGAGC) in the FASTX-Toolkit (http://hannonlab.cshl.edu/fastx_toolkit). The

139 resulting reads were assembled using Trinity (Grabherr et al., 2011) with parameters of

140 -min_contig_length 100,-group_pairs_distance 400, and -SS_lib_type RF to generate a

141 non-redundant gene sequence set.

\section{Repetitive sequence and RNA coding gene analysis}

144 A de novo repeat sequence database for VSA_r1.0 was built using RepeatScout (Price et al., 2005)

145 (version 1.0.5). Repetitive sequences in VSA_r1.0 were searched for using RepeatMasker (version 
146 4.0.3) (http://www.repeatmasker.org) based on known repetitive sequences registered in Repbase

147 (Bao et al., 2015) and the de novo repeat libraries. Transfer RNA genes were predicted using

148 tRNAscan-SE (version 1.23) (Chan and Lowe, 2019) with the default parameters, and ribosomal

149 RNA (rRNA) genes were predicted using BLASTN searches with an E-value cutoff of 1E-10, with

150 the Arabidopsis thaliana 18S rRNA (accession number: X16077) and 5.8S and 25S rRNAs

151 (accession number: X52320) used as query sequences.

\section{Protein-coding gene prediction and annotation}

154 Putative protein-coding genes in VSA_r1.0 were identified with a MAKER pipeline (version 2.31.8)

155 (Cantarel et al., 2008) including ab-initio-, evidence-, and homology-based gene prediction methods.

156 For this prediction, the non-redundant gene sequence set generated from the RNA-Seq analysis and

157 peptide sequences predicted in the genomes of four Fabaceae members, namely, Arachis duranensis

158 (V14167.a1.M1) (Bertioli et al., 2016), Lotus japonicus (rel. 3.0) (Sato et al., 2008), Medicago

159 truncatula (4.0v1) (Young et al., 2011), and Phaseolus vulgaris (v1.0) (Schmutz et al., 2014), were

160 used as a training data set. In addition, BRAKER1 (version 1.3) (Hoff et al., 2016) was used to

161 complete the gene set for VSA_r1.0. Genes related to transposable elements (TEs) were detected

162 using BLASTP searches against the NCBI non-redundant (nr) protein database with an E-value

163 cutoff of 1E-10 and by using InterProScan (version 4.8) (Jones et al., 2014) searches against the

164 InterPro database with an E-value cutoff of 1.0.

165 Putative VSA_r1.0 genes were clustered using CD-hit (version 4.6.1) (Li and Godzik, 2006)

166 with the UniGene set of the four Fabaceae members as above with the parameters $\mathrm{c} \square=\square 0.6$ and aL

$167=0.4$. The predicted genes were annotated with plant gene ontology (GO) slim categories and

168 euKaryotic clusters of Orthologous Groups (KOG) categories (Tatusov et al., 2003), and mapped

169 onto the Kyoto Encyclopedia of Genes and Genomes (KEGG) reference pathways (Ogata et al.,

170 1999). 
Gene expression was quantified by mapping the RNA-Seq reads onto VSA_r1.0 using HISAT2

172 (Kim et al., 2015) followed by normalization to determine fragments per kilobase of exon per

173 million mapped fragments (FPKM) values using StringTie (Pertea et al., 2015) and Ballgown

174 (Frazee et al., 2015) in accordance with the published protocol (Pertea et al., 2016).

176 Genetic diversity analysis

177 Genome-wide sequence variations in wild vetch populations were analyzed by a double-digest

178 restriction-site associated DNA sequencing (ddRAD-Seq) technique (Peterson et al., 2012). In

179 accordance with the workflow established in our previous study (Shirasawa et al., 2016), genomic

180 DNA samples from each line were digested with the restriction enzymes PstI and EcoRI to prepare

181 ddRAD-Seq libraries, which were then sequenced on a HiSeq2000 (Illumina) instrument in

182 paired-end 93 bp mode. Low-quality sequences were removed and adapters were trimmed using

183 PRINSEQ (Schmieder and Edwards, 2011) and fastx_clipper in the FASTX-Toolkit

184 (http://hannonlab.cshl.edu/fastx_toolkit), respectively. The remaining high-quality reads were

185 mapped onto VSA_r1.0 as a reference using Bowtie2 (Langmead and Salzberg, 2012). The resultant

186 sequence alignment-map format (SAM) files were converted to binary sequence alignment-map

187 format (BAM) files and subjected to SNP calling using the mpileup option of SAMtools (Li et al.,

188 2009) and the view option of BCFtools. High-confidence SNPs were selected using VCFtools

189 (Danecek et al., 2011) with the following criteria: (1) depth of coverage $\geq 5$ for each line, (2) SNP

190 quality scores of 999 for each locus, (3) minor allele frequency $\geq 0.05$ for each locus, and (4)

191 proportion of missing data $<0.5$ for each locus. The effects of SNPs on gene function were predicted

192 using SnpEff v4.2 (Cingolani et al., 2012).

193 Nucleotide divergency $(\pi)$ values and heterozygosity levels for SNP sites of each population

194 were calculated using the site-pi and het options in VCFtools (Danecek et al., 2011), respectively.

195 Principal component analysis (PCA) was performed to determine the relationships among samples

196 using TASSEL (Bradbury et al., 2007) and population structure was investigated using 
197 ADMIXTURE (Alexander et al., 2009). The R package WGCNA (Langfelder and Horvath, 2008)

198 was used for SNP module detection.

200 Results

201 Chromosome number of a common vetch line, KSR5

202 A total of 14 chromosomes, including two mini chromosomes, were observed in metaphase cells of 203 root tips of the standard inbred line, KSR5 (Figure 2, Table 1). Relative length of the chromosomes

204 was measured in five cells and sorted by the length order. In accordance with the chromosome length,

205 the 14 chromosomes were grouped into seven pairs (I to VII), suggesting that the genome of KSR5

206 was $2 n=14$. The relative length of the longest chromosome (I) was $22.3 \%$ of the total length of

207 haploid genome, followed by $21.0 \%$ (II), $18.6 \%$ (III), $16.1 \%$ (IV), $10.3 \%$ (V), $9.3 \%$ (VI), and $2.7 \%$

208 (VII).

$210 \quad$ Sequencing and genome assembly

211 The standard inbred line of common vetch ( . sativa), KSR5, was sequenced. In total, 1.8 billion

212 paired-end reads corresponding to $186.7 \mathrm{~Gb}$ (Supplementary Table S2) were obtained. The

213 distribution of distinct $k$-mers $(k=17)$ showed a single main peak at multiplicities of 78 with minor

214 peaks (Figure 3). The size of the common vetch genome was estimated to be 1,769 Mb. The

215 paired-end reads (105× genome coverage) were assembled with mate-pair reads of four libraries

216 (146× genome coverage in total) to obtain 6,487 thousand (k) scaffold sequences of total length 2.5

$217 \mathrm{~Gb}$ with an N50 of $30.5 \mathrm{~kb}$. After removing 6,421 k contaminated sequences and short scaffolds $(<1$

$218 \mathrm{~kb}$ ), sequence gaps presented by Ns in the remaining sequences were filled with PacBio long reads

219 (3× genome coverage) to obtain a draft sequence of the common vetch genome, namely, VSA_r1.0.

220 The total length of VSA_r1.0 was 1,541 Mb and consisted of 54,083 sequences with an N50 of 90.1

$221 \mathrm{~kb}$ (Table 2). Although $513 \mathrm{k}$ gaps occupied $501 \mathrm{Mb}$ in total (32.5\%), the gene space was well

222 represented in accordance with BUSCO examination, indicating 94.1\% ortholog completion. 
Repeat sequence analysis

225 Sequences totaling $782 \mathrm{Mb}(51.9 \%)$ were identified as repeat elements such as transposons and

228 the previously reported repeats, long terminal repeat retroelements were predominant $(200 \mathrm{Mb})$.

229 Furthermore, 109,151 simple-sequence repeats with 52,874 di-, 39,198 tri-, 12,354 tetra-, 3,414

230 penta-, and 1,311 hexa-nucleotide repeat motifs were also found.

Gene prediction and annotation

233 In total, 31,146 protein-encoding genes, with average length of 1,008 bp and N50 of 1,419 bp, were

234 predicted in VSA_r1.0 (Table 2). For the evidence-based MAKER pipeline, 166 million (M) RNA

235 reads from ten tissue samples (Supplementary Table S2) were assembled into 181,211 transcribed

236 sequences and used to predict 27,880 genes (genes with .mk suffix). A further 3,266 genes were

237 predicted using an ab-initio-based method (genes with .br suffix). GO classification assigned 8,878,

2384,059 , and 13,752 genes to the GO slim terms of biological process, cellular component, and

239 molecular function, respectively (Supplementary Table S3). KOG analysis revealed 2,766, 4,888,

240 and 4,424 genes with significant similarities to genes involved in information storage and processing,

241 cellular processing and signaling, and metabolism, respectively (Supplementary Table S4). Finally,

2421,720 genes were mapped to KEGG metabolic pathways (Supplementary Table S5). Gene clustering

243 analysis revealed 5,566 gene clusters that were common to the five legume species tested ( $V$. sativa,

244 A. duranensis, L. japonicus, M. truncatula, and P. vulgaris) and 12,321 clusters that were unique to

245 common vetch (Figure 4). In addition to mRNA sequences, 58 rRNA- and 1,437 tRNA-encoding

246 genes were predicted. 
249 Genome-wide SNPs were identified across the 12 common vetch populations from Japan, consisting

250 of 1,243 lines, and eight lines from France, Germany, Greece, Iran, Italy, and Tunisia from the

251 NARO GeneBank (Tsukuba, Japan) (Supplementary Table S1). Approximately 1.1 million

252 ddRAD-Seq reads per sample were obtained (Supplementary Table S2) and $84.4 \%$ of the reads

253 aligned to the VSA_r1.0 reference sequence. The ddRAD-Seq reads covered $2.4 \mathrm{Mb}(0.16 \%)$ of the

254 reference assembly with $\geq 5$ reads. Sequence alignments detected 46,715 high-confidence SNPs

255 (30.9\% transitions and 69.1\% transversions). SNP density was calculated as 1 SNP per $51 \mathrm{bp}$. When

256 only the 12 populations from Japan were considered, the number of SNPs decreased to 24,118 (1

257 SNP per $100 \mathrm{bp}$ ), ranging from 4,709 SNPs in the SDI population (1 SNP per $510 \mathrm{bp}$ ) to 10,040

258 SNPs in the ABK population (1 SNP per 239 bp) (Table 4).

259 PCA and admixture analysis indicated that there were 2-11 subpopulations in each of the 12

260 populations from Japan (Figure 5, Table 3, Supplementary Figures S1). The observed heterozygosity

261 scores were lower than the expected values (Table 4). Nucleotide divergency scores $(\pi)$ at SNP sites

262 were similarly distributed across ten of the populations from Japan, with median values of 0.31-0.34.

263 The remaining two populations, NGT and SDI, exhibited median values of $\sim 0.25$ (Table 4). Of the

26446,715 high-confidence SNPs, 24,118 clustered according to their $\pi$ scores to generate 82 modules

265 (Supplementary Figure S2). Of these, the $\pi$ scores of one cluster, 'cyan', which contained 190 SNPs,

266 negatively correlated with the latitude of sampling location (Figure 1 and 6). In total, 88 genes were

267 associated with the 190 SNPs, and one of the genes (Vsa_sc30698.1_g030.1.mk) showed sequence

268 similarity to the Arabidopsis gene for a MADS-box protein, SUPPRESSOR OF

269 OVEREXPRESSION OF CONSTANS1 (SOC1), known to be involved in the flowering pathway in

270 plants. Vsa_sc30698.1_g030.1.mk was predominantly transcribed in tendrils (FPKM = 5.0) followed

271 by apical buds (0.5) and stems (0.4), whereas no expression was observed in the other seven tissues,

272 i.e., roots, seedlings, immature and mature leaves, flower buds, flowers, and pods.

273

\section{Discussion}


275 A draft common vetch (V. sativa) genome sequence was generated in this study. Although several

276 legume genome sequences were released previously (Bauchet et al., 2019), this is the first report of a

277 genome from the genus Vicia, which contains several agronomically important legume crops such as

278 fava bean (V.faba). Vicia genomes are large (e.g., $1.8 \mathrm{~Gb}$ for $V$. sativa and $13 \mathrm{~Gb}$ for $V$. faba) due to

279 their massive repetitive sequences, including TEs (Bryant and Hughes, 2011; Hill et al., 2005;

280 Nouzova et al., 2001; Pearce et al., 1996), hampering de novo genome assembly in this genus

281 (Bauchet et al., 2019). As might therefore be expected, more than half of the $V$. sativa genome

282 assembly was comprised of repetitive sequences (Table 3). The assembly contained up to 54,083

283 contig sequences and included $513 \mathrm{k}$ gaps occupying $>500 \mathrm{Mb}$ (Table 2). The short-read technology

284 employed for sequencing might therefore be insufficient to span the repeats. Although construction

285 of contiguous sequences from the short reads was challenging, a near complete gene set was

286 successfully identified in the assembly (Table 2). Whereas it was impossible to compare the genome

287 structure of common vetch with those of relatives due to the fragmented genome sequences,

288 clustering analysis of the gene sequences would provide insights into the gene homoeology in

289 legume species (Figure 4). The genome resources developed in this study will be invaluable for

290 forthcoming gene discovery studies, such as transcriptome analysis and allele mining, in Vicia.

291 We reproducibly observed seven pairs of chromosomes (I to VII) in the root-tip cells of KSR5

292 (Figure 2), among of which one pair (VII) was so small occupying only $2.7 \%$ of the total length of

293 the seven chromosome pairs (Table 1). One type of mini chromosomes, so called B chromosomes

294 which are comprised of repetitive sequence, have been reported in numerous groups of plants so far,

295 but the biological function has not been known (Houben, 2017). B chromosomes are not necessary

296 for the growth and normal development of organisms and show non-Mendelian inheritance patterns

297 (Houben, 2017). This could be one of the reasons for the different chromosome numbers in Vicia

298 sativa (Ladizinsky, 1998; Ladizinsky and Waines, 1982; Navratilova et al., 2003). Further

299 chromosome observations and fluorescence in situ hybridization with the repetitive sequences as

300 probes across multiple lines would characterize and identify the mini chromosomes observed in this 
study. Alternatively, sterility of F1 hybrids derived from crosses between plants with different

chromosome numbers should be analyzed to gain insights into the function of the small chromosomes.

Twelve common vetch populations from Japan were examined, each of which contained 2-11 founder plants were limited even in populations grown under natural environmental conditions.

307 Heterozygosity is thought to contribute strongly to the survival of plant populations under natural at the individual level due to self-pollination. This suggested that high heterozygosity at the population level is sufficient to allow adaptation and survival under natural conditions in autogamous

312 common vetch.

313 Human domestication of wild plant species for agriculture involved selection of individual

314 plants with desirable traits (Izawa et al., 2009; Vaughan et al., 2007). More recently, elite cultivars

315 have been developed with enhanced yield performance to satisfy global food requirements (Hickey

316 et al., 2019). The successive selection of small numbers of individual plants during these processes

317 produced severe bottleneck effects and resulted in decreased genetic diversity and lower tolerance to

318 biotic and abiotic stresses (Canc $\square$ ado, 2011). Heterozygosity at specific genome regions was also

319 lost in some wild plants (Figure 6), as reported previously (Mendez-Vigo et al., 2011). This

320 suggested that genome-wide genetic heterogeneity is not necessarily required for plants to survive

321 under natural conditions. Recent studies have proposed de novo-, super-, or neo-domestication

322 (Fernie and Yan, 2019; Hickey et al., 2019; Vaughan et al., 2007), whereby genetic loci for

323 agronomically important traits are introduced to cultivated crop varieties from wild plants. However,

324 the high genetic heterozygosity levels from the wild donor plants should be retained during the

325 development of new crops to avoid the bottleneck effects sustained during historic domestication of

326 crop varieties (Litrico and Violle, 2015). Therefore, we propose that new domestication of wild 
327 plants should retain high heterozygosity at the population level to capitalize on beneficial traits that

328 increase tolerance to abiotic and biotic stresses, but that agronomically important genetic loci should

329 be fixed to maximize crop potential. The resources generated in this study will provide insights into

330 the de novo domestication of wild plants to develop enhanced crop varieties.

332 Supplementary Data

333 Supplementary Table S1 Plant materials.

334 Supplementary Table S2 Genome and transcriptome data.

335 Supplementary Table S3 Number of KOG functions for protein-encoding genes.

336 Supplementary Table S4 Number of genes mapped to KEGG pathways.

337 Supplementary Table S5 Number of GO terms for protein-encoding genes.

338 Supplementary Figure S1 Cross-validation errors for 12 natural populations of Vicia sativa from

339 Japan in admixture analysis.

340 Supplementary Figure S2 Nucleotide diversity of SNP modules across 12 natural populations of

341 Vicia sativa from Japan.

343 Acknowledgments

344 We are grateful to Dr. H. Masumoto (Kazusa DNA Research Institute) for his kind support. We

345 thank T. Fujishiro, K. Kawashima, Y. Kishida, M. Kohara, C. Minami, S. Nakayama, K. Nanri, S.

346 Sasamoto, C. Takahashi, H. Tsuruoka, A. Watanabe, and M. Yamada (Kazusa DNA Research

347 Institute) and staff of the Department of Technical Development at the Institute for Sustainable

348 Agro-ecosystem Services (University of Tokyo) for their technical assistance. Plant materials were

349 provided by the NIAS GeneBank (Tsukuba, Japan). This work was supported by KAKENHI

350 (24710237 and 221S0002) and the Kazusa DNA Research Institute Foundation. 
353 Sequence data are available from the Sequence Read Archive (DRA) of DNA Data Bank of Japan

354 (DDBJ) under accession numbers DRA004347 for whole genome sequencing, DRA004313 for

355 RNA-Seq, and DRA004301-DRA004312 for ddRAD-Seq (Supplementary Table S2). The DDBJ

356 accession numbers of the assembled sequences are BLWO01000001-BLWO01054083. Genome

357 information is available at Plant GARDEN (https://plantgarden.jp). 
Alexander DH, Novembre J, Lange K. 2009. Fast model-based estimation of ancestry in unrelated individuals. Genome Res 19, 1655-1664.

Altschul SF, Gish W, Miller W, Myers EW, Lipman DJ. 1990. Basic local alignment search tool. J Mol Biol 215, 403-410.

364 Bao W, Kojima KK, Kohany O. 2015. Repbase Update, a database of repetitive elements in 365 eukaryotic genomes. Mob DNA 6, 11.

366 Bauchet GJ, Bett KE, Cameron CT, Campbell JD, Cannon EKS, Cannon SB, Carlson JW, 367 Chan A, Cleary A, Close TJ, Cook DR, Cooksey AM, Coyne C, Dash S, Dickstein R, Farmer 368 AD, Fernández $\square$ Baca D, Hokin S, Jones ES, Kang Y, Monteros MJ, Muñoz $\square$ Amatriaín M, Mysore KS, Pislariu CI, Richards C, Shi A, Town CD, Udvardi M, Wettberg EB, Young ND, Zhao PX. 2019. The future of legume genetic data resources: Challenges, opportunities, and priorities. Legume Science 1.

372 Bertioli DJ, Cannon SB, Froenicke L, Huang G, Farmer AD, Cannon EK, Liu X, Gao D, 373 Clevenger J, Dash S, Ren L, Moretzsohn MC, Shirasawa K, Huang W, Vidigal B, Abernathy B, 374 Chu Y, Niederhuth CE, Umale P, Araujo AC, Kozik A, Kim KD, Burow MD, Varshney RK, 375 Wang X, Zhang X, Barkley N, Guimaraes PM, Isobe S, Guo B, Liao B, Stalker HT, Schmitz RJ, Scheffler BE, Leal-Bertioli SC, Xun X, Jackson SA, Michelmore R, Ozias-Akins P. 2016. The genome sequences of Arachis duranensis and Arachis ipaensis, the diploid ancestors of cultivated peanut. Nat Genet 48, 438-446. software for association mapping of complex traits in diverse samples. Bioinformatics $\mathbf{2 3}$, 2633-2635.

Bryant JA, Hughes SG. 2011. Vicia. In: Kole C, ed. Wild Crop Relatives: Genomic and Breeding Resources. Berlin Heidelberg: Springer-Verlag, 273-289. Canc $\square$ ado G. 2011. The Importance of Genetic Diversity to Manage Abiotic Stress. In: Shanker A, ed. Abiotic Stress in Plants - Mechanisms and Adaptations: InTech, 351-366.

386 Cantarel BL, Korf I, Robb SM, Parra G, Ross E, Moore B, Holt C, Sanchez Alvarado A, 387 Yandell M. 2008. MAKER: an easy-to-use annotation pipeline designed for emerging model 388 organism genomes. Genome Res 18, 188-196.

389 Chan PP, Lowe TM. 2019. tRNAscan-SE: Searching for tRNA Genes in Genomic Sequences.

390 Methods Mol Biol 1962, 1-14.

391 Cingolani P, Platts A, Wang le L, Coon M, Nguyen T, Wang L, Land SJ, Lu X, Ruden DM.

392 2012. A program for annotating and predicting the effects of single nucleotide polymorphisms, 393 SnpEff: SNPs in the genome of Drosophila melanogaster strain w1118; iso-2; iso-3. Fly (Austin) 6, $39480-92$. 
Danecek P, Auton A, Abecasis G, Albers CA, Banks E, DePristo MA, Handsaker RE, Lunter G, Marth GT, Sherry ST, McVean G, Durbin R, Genomes Project Analysis G. 2011. The variant call format and VCFtools. Bioinformatics 27, 2156-2158.

398 English AC, Richards S, Han Y, Wang M, Vee V, Qu J, Qin X, Muzny DM, Reid JG, Worley KC, Gibbs RA. 2012. Mind the gap: upgrading genomes with Pacific Biosciences RS long-read sequencing technology. PLoS One 7, e47768. Fernie AR, Yan J. 2019. De Novo Domestication: An Alternative Route toward New Crops for the Future. Mol Plant 12, 615-631.

Frazee AC, Pertea G, Jaffe AE, Langmead B, Salzberg SL, Leek JT. 2015. Ballgown bridges the gap between transcriptome assembly and expression analysis. Nat Biotechnol 33, 243-246.

Fu YB. 2015. Understanding crop genetic diversity under modern plant breeding. Theor Appl Genet 128, 2131-2142.

407 Grabherr MG, Haas BJ, Yassour M, Levin JZ, Thompson DA, Amit I, Adiconis X, Fan L, 408 Raychowdhury R, Zeng Q, Chen Z, Mauceli E, Hacohen N, Gnirke A, Rhind N, di Palma F, 409 Birren BW, Nusbaum C, Lindblad-Toh K, Friedman N, Regev A. 2011. Full-length transcriptome assembly from RNA-Seq data without a reference genome. Nat Biotechnol 29, correction through iterative short read consensus. Bioinformatics 30, 3004-3011.

414 Hickey LT, A NH, Robinson H, Jackson SA, Leal-Bertioli SCM, Tester M, Gao C, Godwin ID, 415 Hayes BJ, Wulff BBH. 2019. Breeding crops to feed 10 billion. Nat Biotechnol 37, 744-754.

416 Hill P, Burford D, Martin DM, Flavell AJ. 2005. Retrotransposon populations of Vicia species 417 with varying genome size. Mol Genet Genomics 273, 371-381.

418 Hoff KJ, Lange S, Lomsadze A, Borodovsky M, Stanke M. 2016. BRAKER1: Unsupervised 419 RNA-Seq-Based Genome Annotation with GeneMark-ET and AUGUSTUS. Bioinformatics 32, $420 \quad 767-769$.

421 Houben A. 2017. B Chromosomes - A Matter of Chromosome Drive. Front Plant Sci 8, 210.

422 Izawa T, Konishi S, Shomura A, Yano M. 2009. DNA changes tell us about rice domestication.

423 Curr Opin Plant Biol 12, 185-192.

424 Jones P, Binns D, Chang HY, Fraser M, Li W, McAnulla C, McWilliam H, Maslen J, Mitchell 425 A, Nuka G, Pesseat S, Quinn AF, Sangrador-Vegas A, Scheremetjew M, Yong SY, Lopez R, 426 Hunter S. 2014. InterProScan 5: genome-scale protein function classification. Bioinformatics 30 , 427 1236-1240.

428 Kim D, Langmead B, Salzberg SL. 2015. HISAT: a fast spliced aligner with low memory 429 requirements. Nat Methods 12, 357-360.

430 Ladizinsky G. 1998. Plant Evolution under Domestication. Netherlands: Kluwer Academic 431 Publishers. 
Ladizinsky G, Waines G. 1982. Seed protein polymorphism inVicia sativa agg. (Fabaceae). Plant

Systematics and Evolution 141, 1-5.

434 Langfelder P, Horvath S. 2008. WGCNA: an R package for weighted correlation network analysis.

435 BMC Bioinformatics 9, 559.

436 Langmead B, Salzberg SL. 2012. Fast gapped-read alignment with Bowtie 2. Nat Methods 9 ,

$437 \quad 357-359$.

438 Li H, Handsaker B, Wysoker A, Fennell T, Ruan J, Homer N, Marth G, Abecasis G, Durbin R, 439 Genome Project Data Processing S. 2009. The Sequence Alignment/Map format and SAMtools.

440 Bioinformatics 25, 2078-2079.

441 Li W, Godzik A. 2006. Cd-hit: a fast program for clustering and comparing large sets of protein or

442 nucleotide sequences. Bioinformatics 22, 1658-1659.

443 Litrico I, Violle C. 2015. Diversity in Plant Breeding: A New Conceptual Framework. Trends Plant 444 Sci 20, 604-613.

445 Luo R, Liu B, Xie Y, Li Z, Huang W, Yuan J, He G, Chen Y, Pan Q, Liu Y, Tang J, Wu G, 446 Zhang H, Shi Y, Liu Y, Yu C, Wang B, Lu Y, Han C, Cheung DW, Yiu SM, Peng S, Xiaoqian

447 Z, Liu G, Liao X, Li Y, Yang H, Wang J, Lam TW, Wang J. 2012. SOAPdenovo2: an

448 empirically improved memory-efficient short-read de novo assembler. Gigascience 1, 18.

449 Mammadov J, Buyyarapu R, Guttikonda SK, Parliament K, Abdurakhmonov IY, Kumpatla

450 SP. 2018. Wild Relatives of Maize, Rice, Cotton, and Soybean: Treasure Troves for Tolerance to

451 Biotic and Abiotic Stresses. Front Plant Sci 9, 886.

452 Marcais G, Kingsford C. 2011. A fast, lock-free approach for efficient parallel counting of

453 occurrences of k-mers. Bioinformatics 27, 764-770.

454 Mendez-Vigo B, Pico FX, Ramiro M, Martinez-Zapater JM, Alonso-Blanco C. 2011. Altitudinal

455 and climatic adaptation is mediated by flowering traits and FRI, FLC, and PHYC genes in

456 Arabidopsis. Plant Physiol 157, 1942-1955.

457 Mundt CC. 2002. Use of multiline cultivars and cultivar mixtures for disease management. Annu

458 Rev Phytopathol 40, 381-410.

459 Navratilova A, Neumann P, Macas J. 2003. Karyotype analysis of four Vicia species using in situ

460 hybridization with repetitive sequences. Ann Bot 91, 921-926.

461 Nouzova M, Neumann P, Navratilova A, Galbraith DW, Macas J. 2001. Microarray-based

462 survey of repetitive genomic sequences in Vicia spp. Plant Mol Biol 45, 229-244.

463 Ogata H, Goto S, Sato K, Fujibuchi W, Bono H, Kanehisa M. 1999. KEGG: Kyoto Encyclopedia

464 of Genes and Genomes. Nucleic Acids Res 27, 29-34.

465 Pearce SR, Harrison G, Li D, Heslop-Harrison J, Kumar A, Flavell AJ. 1996. The Ty1-copia

466 group retrotransposons in Vicia species: copy number, sequence heterogeneity and chromosomal 467 localisation. Mol Gen Genet 250, 305-315.

468 Pertea M, Kim D, Pertea GM, Leek JT, Salzberg SL. 2016. Transcript-level expression analysis 469 of RNA-seq experiments with HISAT, StringTie and Ballgown. Nat Protoc 11, 1650-1667. 

enables improved reconstruction of a transcriptome from RNA-seq reads. Nat Biotechnol 33,

472 290-295.

473 Peterson BK, Weber JN, Kay EH, Fisher HS, Hoekstra HE. 2012. Double digest RADseq: an inexpensive method for de novo SNP discovery and genotyping in model and non-model species.

475 PLoS One 7, e37135.

476 Price AL, Jones NC, Pevzner PA. 2005. De novo identification of repeat families in large genomes. 477 Bioinformatics 21 Suppl 1, i351-358.

478 Sato S, Nakamura Y, Kaneko T, Asamizu E, Kato T, Nakao M, Sasamoto S, Watanabe A, Ono

479 A, Kawashima K, Fujishiro T, Katoh M, Kohara M, Kishida Y, Minami C, Nakayama S, Nakazaki N, Shimizu Y, Shinpo S, Takahashi C, Wada T, Yamada M, Ohmido N, Hayashi M, Fukui K, Baba T, Nakamichi T, Mori H, Tabata S. 2008. Genome structure of the legume, Lotus japonicus. DNA Res 15, 227-239.

483 Schmieder R, Edwards R. 2011. Quality control and preprocessing of metagenomic datasets.

484 Bioinformatics 27, 863-864.

485 Schmutz J, McClean PE, Mamidi S, Wu GA, Cannon SB, Grimwood J, Jenkins J, Shu S, Song

486 Q, Chavarro C, Torres-Torres M, Geffroy V, Moghaddam SM, Gao D, Abernathy B, Barry K, 487 Blair M, Brick MA, Chovatia M, Gepts P, Goodstein DM, Gonzales M, Hellsten U, Hyten DL, 488 Jia G, Kelly JD, Kudrna D, Lee R, Richard MM, Miklas PN, Osorno JM, Rodrigues J, 489 Thareau V, Urrea CA, Wang M, Yu Y, Zhang M, Wing RA, Cregan PB, Rokhsar DS, Jackson 490 SA. 2014. A reference genome for common bean and genome-wide analysis of dual domestications. 491 Nat Genet 46, 707-713.

492 Schneider CA, Rasband WS, Eliceiri KW. 2012. NIH Image to ImageJ: 25 years of image analysis. 493 Nat Methods 9, 671-675.

494 Shirasawa K, Hirakawa H, Isobe S. 2016. Analytical workflow of double-digest restriction 495 site-associated DNA sequencing based on empirical and in silico optimization in tomato. DNA Res 496 23, 145-153.

497 Simao FA, Waterhouse RM, Ioannidis P, Kriventseva EV, Zdobnov EM. 2015. BUSCO:

498 assessing genome assembly and annotation completeness with single-copy orthologs. Bioinformatics 499 31, 3210-3212.

500 Tatusov RL, Fedorova ND, Jackson JD, Jacobs AR, Kiryutin B, Koonin EV, Krylov DM, 501 Mazumder R, Mekhedov SL, Nikolskaya AN, Rao BS, Smirnov S, Sverdlov AV, Vasudevan S, 502 Wolf YI, Yin JJ, Natale DA. 2003. The COG database: an updated version includes eukaryotes. 503 BMC Bioinformatics 4, 41.

504 Vaughan DA, Balazs E, Heslop-Harrison JS. 2007. From crop domestication to 505 super-domestication. Ann Bot 100, 893-901.

506 Young ND, Debelle F, Oldroyd GE, Geurts R, Cannon SB, Udvardi MK, Benedito VA, Mayer 507 KF, Gouzy J, Schoof H, Van de Peer Y, Proost S, Cook DR, Meyers BC, Spannagl M, Cheung 
508 F, De Mita S, Krishnakumar V, Gundlach H, Zhou S, Mudge J, Bharti AK, Murray JD,

509 Naoumkina MA, Rosen B, Silverstein KA, Tang H, Rombauts S, Zhao PX, Zhou P, Barbe V,

510 Bardou P, Bechner M, Bellec A, Berger A, Berges H, Bidwell S, Bisseling T, Choisne N,

511 Couloux A, Denny R, Deshpande S, Dai X, Doyle JJ, Dudez AM, Farmer AD, Fouteau S,

512 Franken C, Gibelin C, Gish J, Goldstein S, Gonzalez AJ, Green PJ, Hallab A, Hartog M, Hua

513 A, Humphray SJ, Jeong DH, Jing Y, Jocker A, Kenton SM, Kim DJ, Klee K, Lai H, Lang C,

514 Lin S, Macmil SL, Magdelenat G, Matthews L, McCorrison J, Monaghan EL, Mun JH, Najar

515 FZ, Nicholson C, Noirot C, O'Bleness M, Paule CR, Poulain J, Prion F, Qin B, Qu C, Retzel EF,

516 Riddle C, Sallet E, Samain S, Samson N, Sanders I, Saurat O, Scarpelli C, Schiex T, Segurens

517 B, Severin AJ, Sherrier DJ, Shi R, Sims S, Singer SR, Sinharoy S, Sterck L, Viollet A, Wang

518 BB, Wang K, Wang M, Wang X, Warfsmann J, Weissenbach J, White DD, White JD, Wiley

519 GB, Wincker P, Xing Y, Yang L, Yao Z, Ying F, Zhai J, Zhou L, Zuber A, Denarie J, Dixon

520 RA, May GD, Schwartz DC, Rogers J, Quetier F, Town CD, Roe BA. 2011. The Medicago

521 genome provides insight into the evolution of rhizobial symbioses. Nature 480, 520-524.

522 Zhu Y, Chen H, Fan J, Wang Y, Li Y, Chen J, Fan J, Yang S, Hu L, Leung H, Mew TW, Teng

523 PS, Wang Z, Mundt CC. 2000. Genetic diversity and disease control in rice. Nature 406, 718-722. 
525 Table 1 Relative chromosome length of Vicia sativa, KSR5

\begin{tabular}{crc}
\hline Chromosome & Relative length (\%) & S.d. $^{*}$ \\
\hline I & 22.3 & 0.7 \\
II & 21.0 & 0.7 \\
III & 18.6 & 1.3 \\
IV & 16.1 & 1.6 \\
V & 10.3 & 0.7 \\
VI & 9.1 & 0.6 \\
VII & 2.7 & 1.0 \\
\hline
\end{tabular}

526 *Standard deviation $(\mathrm{n}=10)$ 
528 Table 2 Assembly statistics of the common vetch (Vicia sativia) genome assembly VSA_r1.0

\begin{tabular}{ll}
\hline & VSA_r1.0 \\
\hline Number of scaffolds & 54,083 \\
Assembly size (bp) & $1,541,180,487$ \\
Scaffold N50 (bp) & 90,105 \\
Maximal scaffold (bp) & 871,438 \\
Number of gaps & 513,235 \\
Gap size (bp) & $501,483,283$ \\
Complete and single-copy BUSCO & $77.5 \%$ \\
Complete and duplicated BUSCO & $16.6 \%$ \\
Fragmented BUSCO & $2.9 \%$ \\
Missing BUSCO & $2.9 \%$ \\
Number of genes predicted & 31,146 \\
\hline
\end{tabular}


530 Table 3 Repeat sequences in the VSA_r1.0 assembly

\begin{tabular}{lll}
\hline Repeat type & Length occupied (bp) & $\%$ \\
\hline SINEs $^{\mathrm{b}}$ & 85,029 & 0.0 \\
LINEs $^{\mathrm{b}}$ & $10,462,622$ & 0.7 \\
LTR elements $^{\mathrm{b}}$ & $200,723,246$ & 13.0 \\
DNA elements & $15,595,575$ & 1.0 \\
Helitrons & $1,469,970$ & 0.1 \\
Satellites & $17,496,670$ & 1.1 \\
Simple repeats & $17,496,670$ & 1.1 \\
Low complexity $^{4}$ & $4,468,370$ & 0.3 \\
Novel repeats & $531,016,543$ & 34.5 \\
Total $^{\mathrm{a}}$ & $\mathbf{7 8 2 , 8 3 4 , 2 0 1}$ & $\mathbf{5 0 . 8}$ \\
\hline
\end{tabular}

$531{ }^{\mathrm{a}}$ Non-redundant sequence length of the repeats overlapping in the genome.

532 bINEs: short interspersed nuclear elements; LINEs: long interspersed nuclear elements; and LTR:

533 long terminal repeat. 
535 Table 4 Cluster, heterozygosity, and nucleotide diversity calculated from SNPs of 12 common vetch natural populations in Japan

\begin{tabular}{|c|c|c|c|c|c|c|c|}
\hline Population & Sampling location $^{\mathrm{a}}$ & $\begin{array}{l}\text { Number of } \\
\text { individuals }\end{array}$ & $\begin{array}{l}\text { Number of } \\
\text { SNPs }\end{array}$ & $\begin{array}{l}\text { Number of } \\
\text { clusters (K) }\end{array}$ & $\begin{array}{l}\text { Expected } \\
\text { heterozygosity }(\mathrm{He})\end{array}$ & $\begin{array}{l}\text { Observed } \\
\text { heterozygosity (Ho) }\end{array}$ & $\begin{array}{l}\text { Nucleotide } \\
\text { divergency }(\pi)\end{array}$ \\
\hline $\mathrm{ABK}$ & Abiko, Chiba, Japan & 102 & 10,040 & 4 & 0.313 & 0.189 & 0.314 \\
\hline FKO & Fukuoka, Japan & 97 & 9,795 & 7 & 0.318 & 0.057 & 0.319 \\
\hline KGS & Kagoshima, Japan & 109 & 5,189 & 8 & 0.330 & 0.106 & 0.330 \\
\hline KMT & Kimitsu, Chiba, Japan & 95 & 7,256 & 9 & 0.336 & 0.087 & 0.336 \\
\hline KSR & Kisarazu, Chiba, Japan & 88 & 6,450 & 4 & 0.340 & 0.111 & 0.340 \\
\hline KYT & Kyoto, Japan & 104 & 8,974 & 8 & 0.339 & 0.114 & 0.338 \\
\hline KZS & Kazusa, Chiba, Japan & 97 & 7,243 & 4 & 0.334 & 0.147 & 0.334 \\
\hline NGT & Niigata, Japan & 100 & 6,658 & 3 & 0.247 & 0.085 & 0.248 \\
\hline NGY & Nagoya, Aichi, Japan & 102 & 6,891 & 5 & 0.335 & 0.140 & 0.335 \\
\hline OKY & Okayama, Japan & 99 & 9,649 & 11 & 0.337 & 0.085 & 0.336 \\
\hline SDI & Sendai, Miyagi, Japan & 100 & 4,709 & 2 & 0.264 & 0.161 & 0.262 \\
\hline TNS & Tanashi, Tokyo, Japan & 150 & 7,939 & 10 & 0.326 & 0.153 & 0.325 \\
\hline
\end{tabular}

$536{ }^{a}$ Geographical positions are indicated in Figure 1 and Supplementary Table S1. 


\section{Figure Legends}

538 Figure 1 Sampling locations in Japan.

539 Three-letter codes indicate sampling locations in Japan: ABK: Abiko, Chiba; FKO: Fukuoka;

540 KGS: Kagoshima; KMT: Kimitsu, Chiba; KSR: Kisarazu, Chiba; KYT: Kyoto; KZS: Kazusa,

541 Chiba; NGT: Niigata; NGY: Nagoya, Aichi; OKY: Okayama; SDI: Sendai, Miyagi; and

542 TNS: Tanashi, Tokyo.

543 Figure 2 Chromosomes of the common vetch KSR5.

544 Roman numerals indicate chromosome pairs, which order is based on chromosome length (I

545 to VII). Bar $=5 \mu \mathrm{m}$.

546 Figure 3 Genome size estimation for Vicia sativa with the distribution of the number of

547 distinct $k$-mers $(k=17)$ with the given multiplicity values.

548 Figure 4 Venn diagram showing numbers of gene clusters in Vicia sativa and four additional

549 Fabaceae species.

550 Figure 5 Principal component analysis of 12 natural populations of Vicia sativa from Japan.

551 Figure 6 Nucleotide diversity $(\pi)$ of the SNP module 'cyan' (n=190) across 12 natural

552 populations of Vicia sativa in Japan.

553 Three-letter codes indicate sampling locations in Japan: ABK: Abiko, Chiba; FKO: Fukuoka;

554 KGS: Kagoshima; KMT: Kimitsu, Chiba; KSR: Kisarazu, Chiba; KYT: Kyoto; KZS: Kazusa,

555 Chiba; NGT: Niigata; NGY: Nagoya, Aichi; OKY: Okayama; SDI: Sendai, Miyagi; and

556 TNS: Tanashi, Tokyo. 

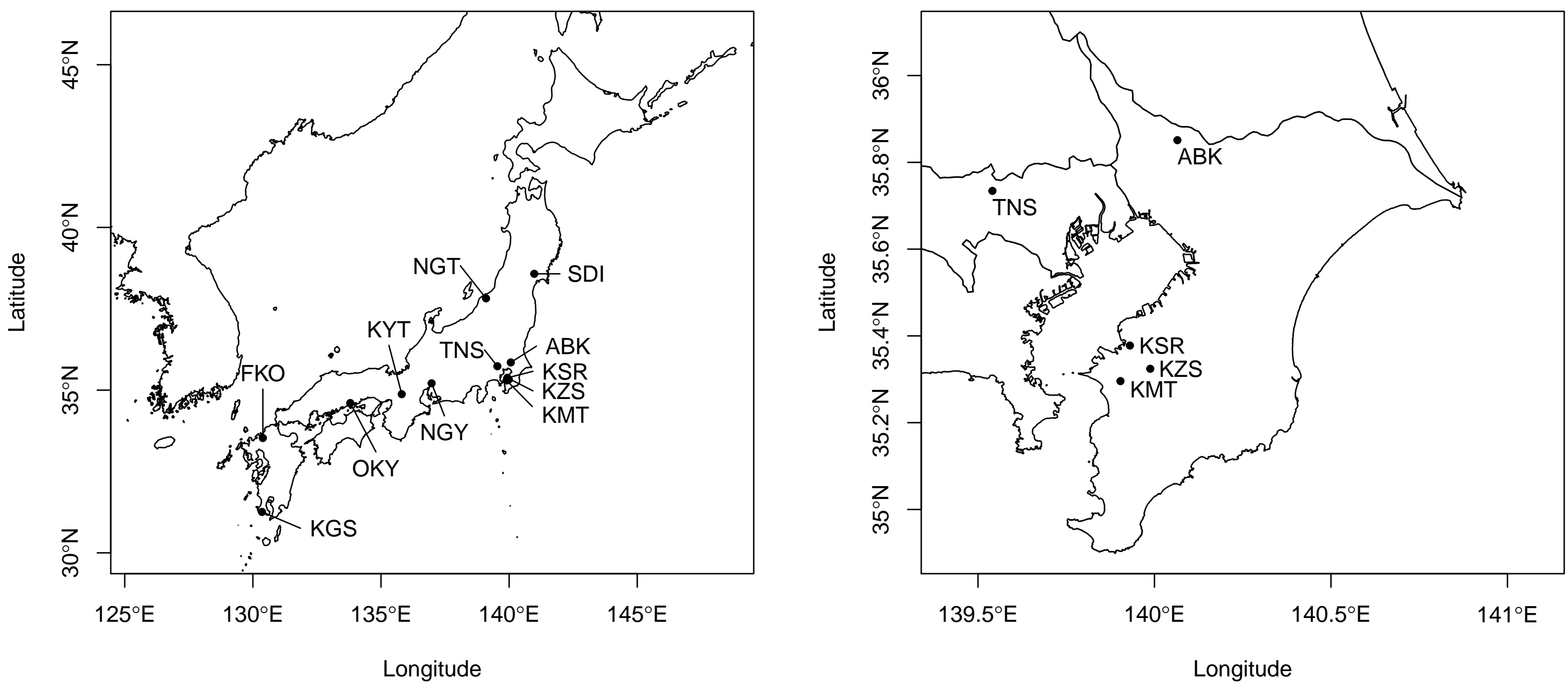
III

II

I

VII

IV

III

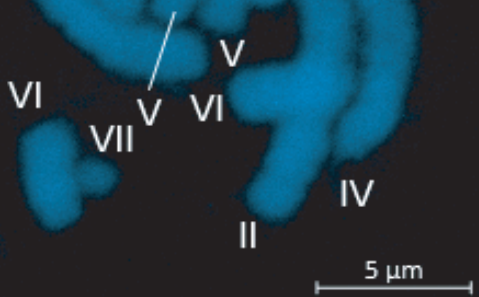




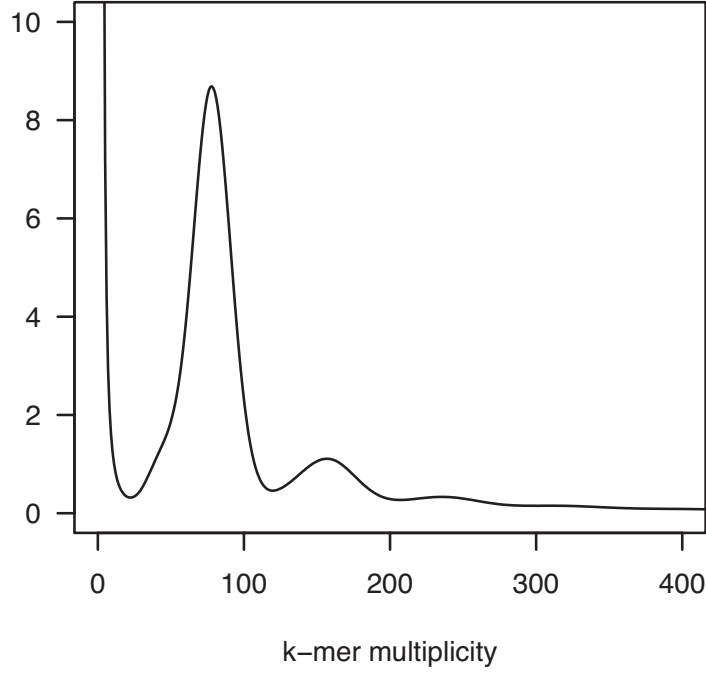


V. sativa

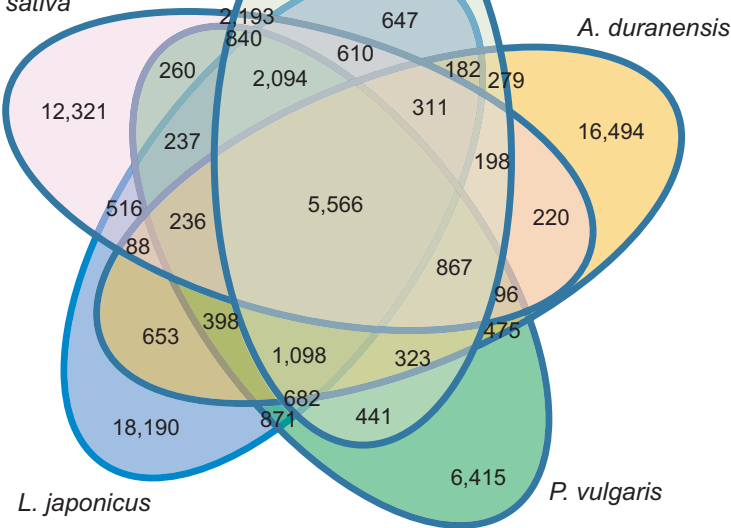


ABK

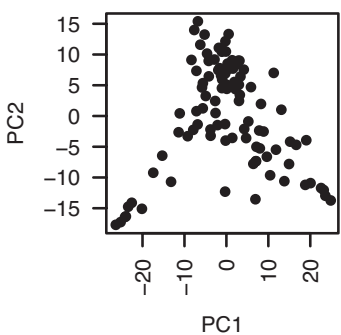

KSR

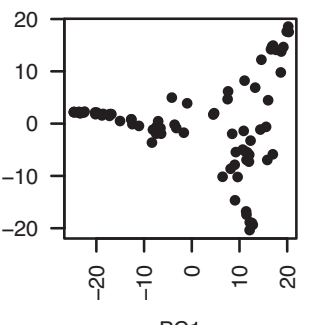

NGY

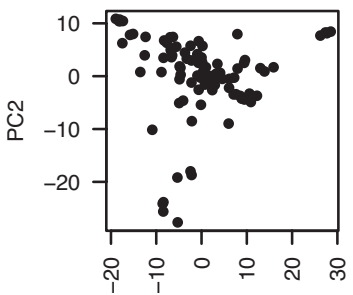

PC1
FKO

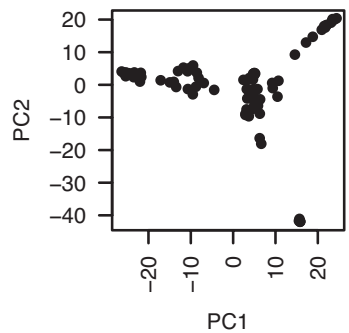

KYT

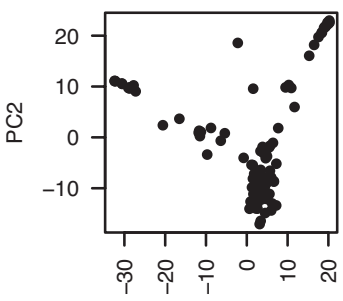

PC1

OKY

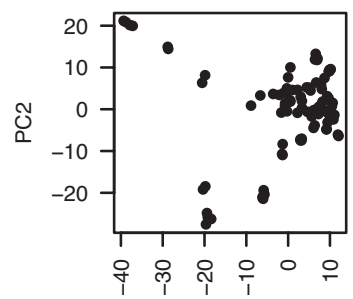

PC1

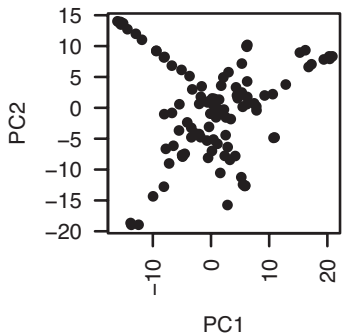

KZS

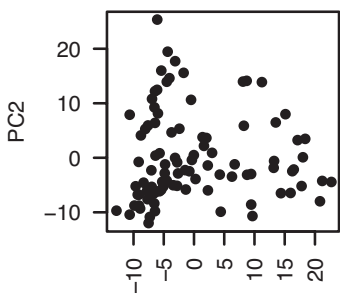

PC1

SDI

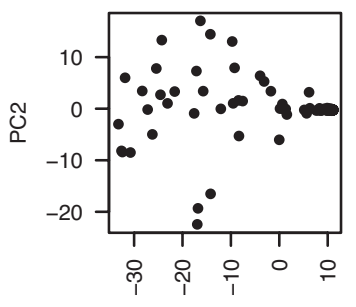

PC1
KMT

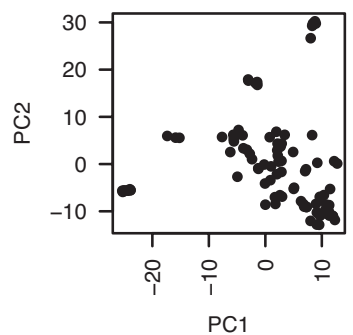

NGT

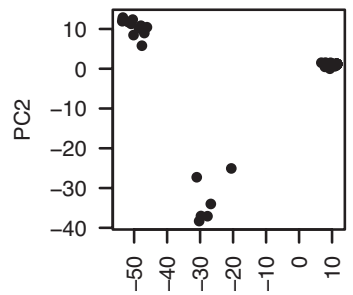

PC1

TNS

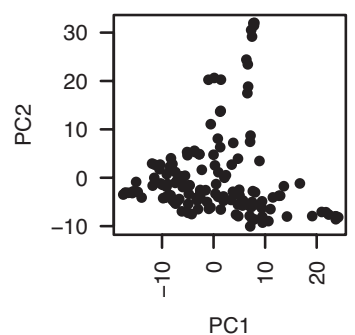




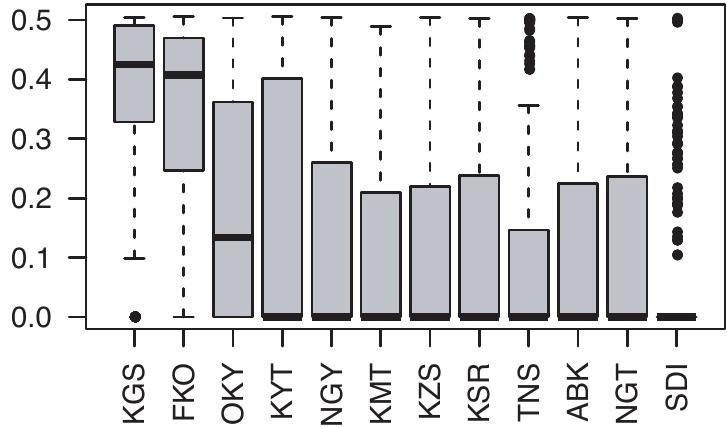

